\title{
Effects of multicomponent exercise on cognitive function in older adults with amnestic mild cognitive impairment: a randomized controlled trial
}

\author{
Takao Suzuki ${ }^{*}$, Hiroyuki Shimada ${ }^{2}$, Hyuma Makizako² ${ }^{2}$ Takehiko Doi ${ }^{2}$, Daisuke Yoshida ${ }^{2}$, Kota Tsutsumimoto ${ }^{2}$, \\ Yuya Anan ${ }^{2}$, Kazuki Uemura ${ }^{2}$, Sangyoon Lee ${ }^{3}$ and Hyuntae Park ${ }^{3}$
}

\begin{abstract}
Background: To examine the effects of a multicomponent exercise program on the cognitive function of older adults with amnestic mild cognitive impairment (aMCl).

Methods: Design: Twelve months, randomized controlled trial; Setting: Community center in Japan; Participants: Fifty older adults (27 men) with aMCl ranging in age from 65 to 93 years (mean age, 75 years); Intervention: Subjects were randomized into either a multicomponent exercise $(n=25)$ or an education control group $(n=25)$. Subjects in the multicomponent exercise group exercised under the supervision of physiotherapists for $90 \mathrm{~min} / \mathrm{d}$, $2 \mathrm{~d} / \mathrm{wk}$, for a total of 80 times over 12 months. The exercises included aerobic exercises, muscle strength training, and postural balance retraining, and were conducted using multiple conditions to stimulate cognitive functions. Subjects in the control group attended three education classes regarding health during the 12-month period. Measurements were administered before, after the 6-month, and after the 12-month intervention period; Measurements: The performance measures included the mini-mental state examination, logical memory subtest of the Wechsler memory scale-revised, digit symbol coding test, letter and categorical verbal fluency test, and the Stroop color word test.
\end{abstract}

Results: The mean adherence to the exercise program was 79.2\%. Improvements of cognitive function following multicomponent exercise were superior at treatment end (group $\times$ time interactions for the mini-mental state examination ( $P=0.04$ ), logical memory of immediate recall $(P=0.03)$, and letter verbal fluency test $(P=0.02)$ ). The logical memory of delayed recall, digit symbol coding, and Stroop color word test showed main effects of time, although there were no group $\times$ time interactions.

Conclusions: This study indicates that exercise improves or supports, at least partly, cognitive performance in older adults with aMCl.

Keywords: Aerobic exercise, MCl, Elderly, Alzheimer's disease, Prevention

\section{Background}

Population-based studies in older adults performed in North America, Europe, and Asia report a prevalence of mild cognitive impairment (MCI) ranging from $11 \%$ to $17 \%$ [1-5], and a prevalence of the amnestic MCI (aMCI) subtype between 3 and 5\% [2,6]. Evidence from both neuropsychological and neuroimaging studies have suggested

\footnotetext{
*Correspondence: suzutaka@ncgg.go.jp

'Research Institute, National Center for Geriatrics and Gerontology, Obu, Japan

Full list of author information is available at the end of the article
}

that $\mathrm{MCI}$ represents a clinical prodrome to degenerative dementias such as Alzheimer's dementia (AD) $[7,8]$. This is particularly the case with aMCI, which is likely to progress to AD [9]. Early treatment of mild to moderate AD is associated with better responses than later treatment [10], so it is conceivable that treating MCI may be particularly effective in delaying the progression to $\mathrm{AD}$.

Clinical aspects have been widely examined as possible biomarkers for $\mathrm{MCI}$ to detect subjects at greater risk of conversion to dementia. Neuropsychological predictors of conversion include performance on specific cognitive

\section{Biomed Central}


tests, particularly those assessing delayed recall and executive functions [11-14]. Reducing or recovering from identified risk factors is important in the prevention of the conversion from $\mathrm{MCI}$ to $\mathrm{AD}$ or to delay the progression of the prodromal symptoms of dementia.

Epidemiological data suggests that moderate exercise and physical activity, such as walking, are associated with a lower risk of dementia [15]. According to these findings, epidemiological studies and randomized controlled trials examining the effects of exercise have proposed it is associated with various cognitive benefits [16-25], and a meta-analysis reported that physical activity or exercise is associated with improvements in attention, processing speed, and executive function in older adults with or without cognitive impairments [26-28]. RCT have been conducted to determine the effects of exercise or physical activity on cognitive functions in older adults with MCI. These studies identified the effects of exercise or physical activity on cognitive function including general cognitive function, executive function and glucometabolic and hypothalamic-pituitary-adrenal axis responses in older adults with MCI $[16,19,22,23]$. However, because the results of these studies differed largely due to differences in methodology, sufficient evidence has not been garnered regarding the relationship between exercise and cognitive function in aMCI.

We designed the present randomized trial to test whether 12 months of supervised multicomponent exercise improved cognitive function among older adults with aMCI. The multicomponent exercise included aerobic exercise, muscle strength training, and postural balance retraining. We adopted the multicomponent regimen because a previous review suggested that participants in combined aerobic and strength training regimens improved cognitive function to a reliably greater degree than those in aerobic training alone $(0.59$ vs. $0.41, \mathrm{SE}=0.043$, $\mathrm{n}=101, \mathrm{p}<0.05)[29]$.

\section{Methods}

\section{Participants}

In this 12-month randomized controlled trial, the subjects were divided into a multicomponent exercise or an education control group at the end of the baseline assessment. Study personnel involved in collection of outcome measures were unaware of each subject's assigned group. The Ethics Committee of the National Center for Geriatrics and Gerontology approved the study protocol. The purpose, nature, and potential risks of the experiments were fully explained to the subjects, and all subjects gave written, informed consent before participating in the study.

Subjects in this study were recruited from our volunteer databases, which included elderly individuals (65 years and over). The inclusion criteria we used were dwelling in the community and being 65 years or age or older. 528 prospective subjects with a Clinical Dementia Rating (CDR) [30] of 0.5, or who complained of memory impairments, were recruited for the initial eligibility assessments. 135 subjects participated in the secondary eligibility assessments, including neuropsychological tests and magnetic resonance imaging. 35 of these 135 subjects were excluded and the remaining 100 subjects met the definition of MCI using the Petersen criteria [31]. The objective memory impairment was defined as having a lower memory in the Logical Memory II subtest of the Wechsler memory scale-revised (WMS-LM II) [32]. The cut-off score to define the aMCI was adjusted by educational history ( $0-9$ years: $<7$ points, $10-15$ years: $<10$ points, more than 16 years: $<12$ points) [33]. The exclusion criteria we used included having a $\mathrm{CDR}=0,1,2$, and 3 , a history of neurological, psychiatric, and cardiac disorders or other severe health issues, use of donepezil, loss of independence in basic activities of daily living (ADL), and current participation in other research projects. A diagram consistent with the Consolidated Standards of Reporting Trials (CONSORT) [34] that outlines the subject flow from first contact to study completion is shown in Figure 1. Fifty older adults (mean age $76.0 \pm 7.1$ years, range 65-92 years, male $=27(54 \%)$, mean education level $10.9 \pm 2.5$ years) with aMCI who had objective memory disabilities and who completed the neuroimaging assessments were selected as the subjects in the study.

\section{Intervention}

Subjects in the multicomponent exercise group exercised under the supervision of physiotherapists for $90 \mathrm{~min} / \mathrm{d}, 2 \mathrm{~d} / \mathrm{wk}$, for a total of 80 times over 12 months. Two physiotherapists involved in geriatric rehabilitation and three well-trained instructors conducted each intervention. The exercise class consisted of 16-17 participants, and each supervised session began with a 10-min warm-up period, followed by 20 min of muscle strength exercise. The subjects then practiced aerobic exercise, postural balance retraining, and dual-task training for $60 \mathrm{~min}$. In the aerobic exercises and postural balance retraining, subjects completed circuit training including stair stepping, endurance walking, and walking on balance boards. The mean intensity of the aerobic exercises was approximately $60 \%$ of maximum heart rate. Heart rate was monitored after aerobic exercise each session to take their pulse. One out of every four classes during the intervention period included outdoor walking during approximately 20-30 minutes. These exercises or training were also conducted under multitask conditions. For example, the subjects in the exercise group were asked to invent their own poem while walking. In the ladder training, subjects memorized a step pattern in 


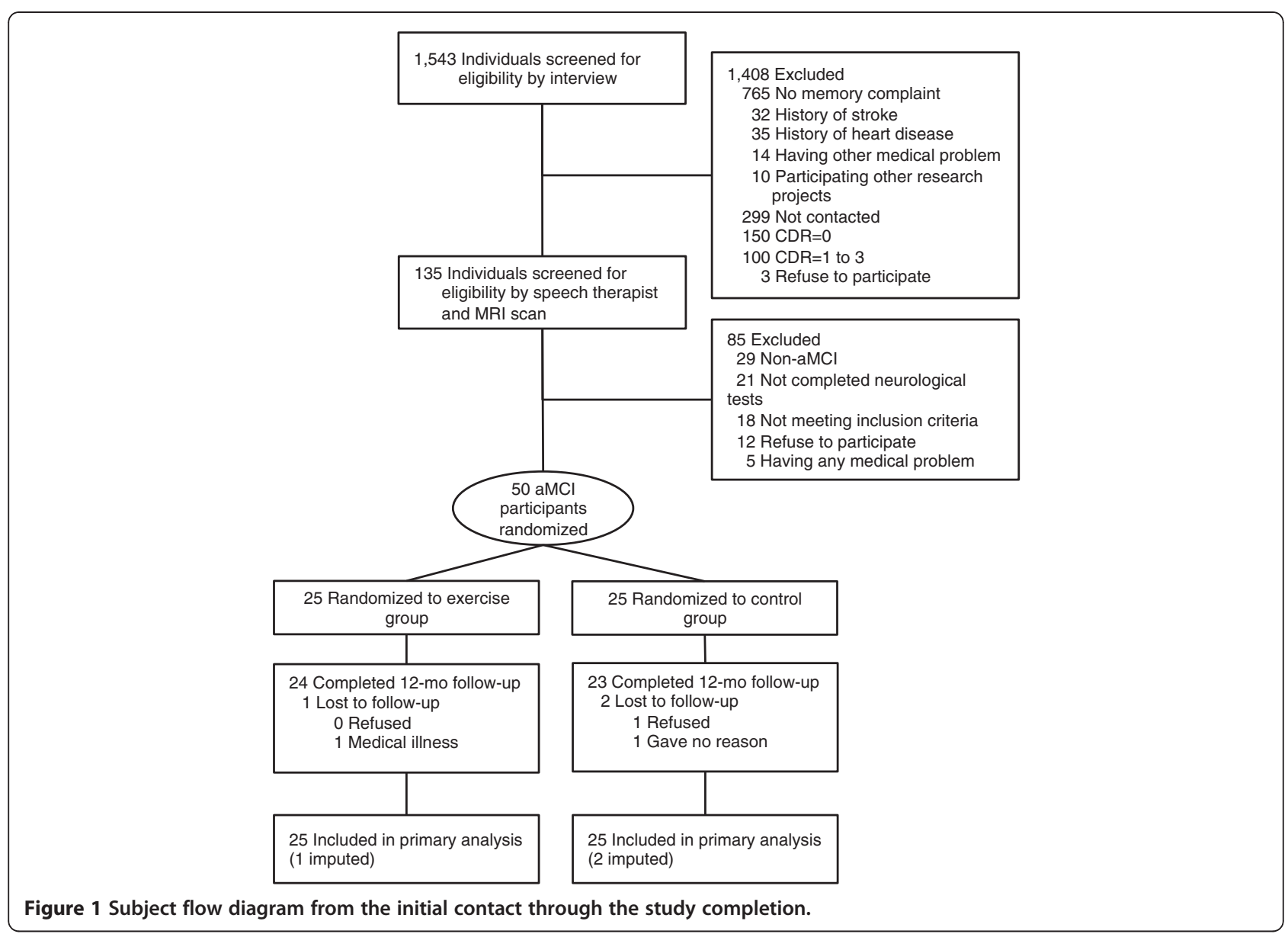

consecutive square segments, and were instructed to step as quickly and accurately as possible. Before and after each session of the program, physiotherapists conducted a health check of each subject. The physiotherapists and well-trained instructors provided ongoing safety monitoring to prevent adverse accidents such as falling during the program. Daily home-based exercise in addition to structured program and outdoor walking was recommended to the exercise group. The subjects allocated the exercise group were asked to recording the amount of time spent on daily home-based exercise and the daily total steps for pedometer in a notebook. The subjects made graphs from the records of amount of time and steps to promote active lifestyle through a self-monitoring. Attendance at each session was recorded and a transportation service was provided for the participants, if necessary, to help the subjects maintain their participation in the program.

Subjects in the education control group attended three education classes regarding health promotion during the 12-month study period. The class provided information regarding aging, healthy diet, oral care, brain image diagnosis, prevention of urinary incontinence, and health checks. However, the group did not receive specific information regarding exercise, physical activity, or cognitive health.

\section{Outcomes}

The cognitive function before, after 6-month, and after 12-month intervention period was measured by speech therapists. Prior to the commencement of the study, all staff received training from the authors in the correct protocols for administering all of the assessment measures included in the study. The assessors introduced and demonstrated the assessments to facilitate understanding of the tests by the participants before they conducted the tests. A speech therapist calculated all of the results of the cognitive function tests.

The mini-mental state examination (MMSE) was measured as a general cognitive function [35]. The WMSLM I and II [32] was used to assess logical memory function. In the WMS-LM, two short stories (story A and B) were read aloud to the subjects, who were then instructed to recall details of the stories immediately (WMS-LM I) and after $30 \mathrm{~min}$ (WMS-LM II) (total recall score $=50)$ [32]. The digit symbol-coding (DSC) subset of the Wechsler Adult Intelligence Scale III was used to assess processing speed [36]. Verbal fluency 
$[37,38]$ was measured by the number of words generated across 60-second trials [37]. The subject's listed words began with a letter composed of Japanese characters for the three trials (letter verbal fluency test: LVFT) and that belonged to a semantic category (category verbal fluency test: CVFT). The total number of correct responses was used for the analysis. The Stroop Color and Word Test (SCWT) [39] was used to assess executive function. For the Stroop test, we used two conditions. First, the subjects were instructed to read out words printed in black ink (e.g., blue) (SCWT-I). Second, they were shown a page with color words printed in incongruent colored inks (e.g., the word blue printed in red ink). The subjects were asked to name the ink color in which the words were printed (while ignoring the word itself) (SCWT-III). There were 24 trials for each condition, and we recorded the time the participants took to read each condition.

\section{Analysis}

The statistical analyses were conducted using SPSS software (Version 20; SPSS Inc., Chicago, IL, USA). The independent samples $t$-test or Chi-square test was used to compare the basic characteristics between the exercise and education control group. The effect of intervention (exercise vs control) over time on the cognitive functions was investigated in intention-to-treat (ITT) analyses. Measurements were analyzed using linear mixed models; analyses assumed missing at random with missingness allowed to be driven by variables included in the analyses. All models included random intercepts to account for correlations between the repeated measures for each participant. The fixed components of the models included effects of group and time and a group $\times$ time interaction. To assess the presence of a group and time effect, where this may change over time, we first determined the existence of time, group, and group $\times$ time interactions. The post hoc analyses were made between times and groups using the Bonferroni method. All statistical significance tests were two-sided, and an alpha-level of 0.05 was considered statistically significant.

\section{Results}

\section{Adherence to intervention}

There were no significant differences in the baseline characteristics between the exercise and education control groups (Table 1). Figure 1 shows the flow of the participants from the time of screening to study completion at 12 months. 47 (exercise group, $\mathrm{n}=24$ ) subjects completed the 12-month follow-up. Two of the twenty-five subjects in the exercise group (1 man and 1 woman) did not attend a single session, but were included in the following analyses. The mean adherence to the exercise program, including these subjects, was $79.2 \%$, and 17
Table 1 Baseline characteristics of the subjects

\begin{tabular}{|c|c|c|}
\hline Characteristic & Exercise $(n=25)$ & Control $(n=25)$ \\
\hline Age, mean $\pm S D$, y & $75.3 \pm 7.5$ & $76.8 \pm 6.8$ \\
\hline Men, No. (\%), n & $13(52.0)$ & $14(56.0)$ \\
\hline Educational level, mean $\pm S D$, y & $11.1 \pm 2.4$ & $10.8 \pm 2.7$ \\
\hline \multicolumn{3}{|l|}{ Blood pressure, mean $\pm \mathrm{SD}, \mathrm{mmHg}$} \\
\hline Diastolic & $77.3 \pm 11.1$ & $74.3 \pm 10.1$ \\
\hline Systolic & $152.2 \pm 21.0$ & $143.7 \pm 21.3$ \\
\hline \multicolumn{3}{|l|}{ Diagnosis, No. (\%), n } \\
\hline Hyper tension $\left(1^{*}\right)$ & $13(52.0)$ & $11(44.0)$ \\
\hline Heart disease $\left(1^{*}\right)$ & $2(8.0)$ & $0(0)$ \\
\hline Diabetes Mellitus & $5(20.0)$ & $3(12.0)$ \\
\hline Medication, 3 and over & $10(40.0)$ & $11(44.0)$ \\
\hline \multicolumn{3}{|l|}{ Blood test, mean \pm SD } \\
\hline Total cholesterol, mg/dL & $212.6 \pm 36.9$ & $202.8 \pm 32.2$ \\
\hline Triglyceride, mg/dL & $146.8 \pm 73.7$ & $130.4 \pm 112.3$ \\
\hline Glucose, mg/dL & $116.3 \pm 27.1$ & $110.4 \pm 23.4$ \\
\hline $\mathrm{HA} 1 \mathrm{C}, \%$ & $5.6 \pm 0.6$ & $5.4 \pm 0.5$ \\
\hline \multicolumn{3}{|l|}{ Cognitive functions, mean \pm SD } \\
\hline MMSE, score & $26.8 \pm 1.8$ & $26.6 \pm 1.6$ \\
\hline WMS-LM I, score & $12.5 \pm 5.9$ & $12.0 \pm 4.9$ \\
\hline WMS-LM II, score & $8.2 \pm 5.4$ & $6.9 \pm 5.0$ \\
\hline DSC, s & $47.5 \pm 15.4$ & $44.3 \pm 16.3$ \\
\hline LVFT, score & $16.0 \pm 5.3$ & $16.9 \pm 6.0$ \\
\hline CVFT, score & $33.1 \pm 6.9$ & $31.2 \pm 7.7$ \\
\hline SCWT-I, S & $22.6 \pm 9.7$ & $23.4 \pm 11.1$ \\
\hline SCWT-III, S & $42.0 \pm 13.7$ & $41.5 \pm 17.7$ \\
\hline \multicolumn{3}{|l|}{ TMIG index, mean $\pm S D$, score } \\
\hline IADL & $5.0 \pm 0.2$ & $4.9 \pm 0.3$ \\
\hline Intellectual activity & $3.8 \pm 0.4$ & $3.8 \pm 0.4$ \\
\hline Social role & $3.6 \pm 0.9$ & $3.6 \pm 0.8$ \\
\hline Total & $12.3 \pm 1.1$ & $12.3 \pm 0.9$ \\
\hline GDS, mean $\pm S D$, score & $3.0 \pm 2.1$ & $2.6 \pm 2.0$ \\
\hline
\end{tabular}

SD; standard deviation, MMSE; mini-mental state examination, WMS-LM II; Logical Memory II subtest of the Wechsler memory scale-revised, LVFT; letter verbal fluency test, CVFT; category verbal fluency test, DSC; digit-symbol cording, SCWT; Stroop Color and Word Test, TMIG index; Tokyo Metropolitan Institute of Gerontology index, GDS; Geriatric Depression Scale. " missing value.

subjects $(68.0 \%)$ in the exercise group attended our intervention program with more than $80 \%$ adherence.

\section{Changes in cognitive function}

Table 2 shows changes in cognitive scores over the 12 months across the groups. On the MMSE, there was a group $\times$ time interaction $(P=0.04)$ indicated benefit of the exercise over time (Figure 2). Although there were no main effects of group or time, the control group showed significant decline in the MMSE score after 6 
Table 2 Comparison of cognitive functions between exercise and control groups

\begin{tabular}{|c|c|c|c|c|c|c|c|c|c|c|c|c|c|}
\hline & \multicolumn{2}{|c|}{$\begin{array}{c}\text { Mean difference }(95 \% \mathrm{Cl}) \text { between before and } \\
\text { after } 6 \text { months }\end{array}$} & \multicolumn{2}{|c|}{$\begin{array}{l}\text { Mean difference }(95 \% \mathrm{Cl}) \text { between before and } \\
\text { after } 12 \text { months }\end{array}$} & \multicolumn{3}{|c|}{ Time } & \multicolumn{3}{|c|}{ Group } & \multicolumn{3}{|c|}{ Group $\times$ time } \\
\hline & Exercise $(n=25)$ & Control $(n=25)$ & Exercise $(n=25)$ & Control $(n=25)$ & df & F value & $P$ value & df & F value & $P$ value & df & $F$ value & $P$ value \\
\hline MMSE & $0.32(-0.96-1.60)$ & $-1.37(-2.66--0.07)$ & $-0.47(-1.75-0.81)$ & $-0.44(-1.74-0.86)$ & 92.1 & 1.17 & $0.32^{d}$ & 48.0 & 2.2 & $0.14^{9}$ & 92.1 & 3.4 & 0.04 \\
\hline WMS-LM I & $3.83(1.40-6.25)$ & $0.60(-1.87-3.06)$ & $4.62(2.19-7.05)$ & $5.00(2.53-7.46)$ & 90.9 & 23.0 & $<0.01{ }^{a}, b, e, f$ & 47.8 & 0.9 & $0.34^{9}$ & 90.9 & 3.9 & 0.03 \\
\hline WMS-LM II & $3.79(1.49-6.10)$ & $2.09(-0.25-4.43)$ & $5.13(2.82-7.43)$ & $6.20(3.86-8.54)$ & 91.1 & 35.4 & $<0.01 a, b, e, f$ & 48.1 & 1.1 & 0.30 & 91.1 & 2.1 & 0.13 \\
\hline DSC & $-0.19(-4.20-3.81)$ & $3.27(-0.80-7.34)$ & $3.64(-0.36-7.64)$ & $3.73(-0.34-7.80)$ & 90.4 & 5.0 & $<0.01$ & 48.0 & 0.2 & 0.65 & 90.4 & 1.4 & 0.25 \\
\hline LVFT & $2.87(0.57-5.17)$ & $-0.97(-3.31-1.37)$ & $2.99(0.69-5.30)$ & $1.61(-0.73-3.95)$ & 91.2 & 5.9 & $<0.01$ & 48.3 & 0.3 & 0.59 & 91.2 & 4.1 & 0.02 \\
\hline CVFT & $1.54(-1.27-4.35)$ & $-1.81(-4.67-1.04)$ & $1.33(-1.48-4.14)$ & $-1.61(-4.46-1.25)$ & 91.2 & 0.02 & 0.98 & 48.5 & 3.1 & 0.08 & 91.2 & 2.5 & 0.09 \\
\hline SCWT-I & $-3.30(-7.56-0.96)$ & $-0.98(-5.30-3.34)$ & $-3.68(-7.94-0.58)$ & $-3.20(-7.52-1.12)$ & 91.5 & 3.9 & 0.02 & 47.7 & 0.73 & 0.40 & 91.5 & 0.5 & 0.62 \\
\hline SCWT-III & $-4.70(-12.72-3.33)$ & $0.15(-8.00-8.30)$ & $-2.61(-10.64-5.42)$ & $-4.57(-12.72-3.58)$ & 92.0 & 1.2 & 0.31 & 48.8 & 0.009 & 0.92 & 92.0 & 1.1 & 0.34 \\
\hline
\end{tabular}

MMSE; mini-mental state examination, WMS-LM; Logical Memory subtest of the Wechsler memory scale-revised, DSC; digit-symbol cording, LVFT; letter verbal fluency test, CVFT; category verbal fluency test, SCWT; Stroop Color and Word Test, TMIG; Tokyo Metropolitan Institute of Gerontology index.

${ }^{\mathrm{a}} \mathrm{P}<.05$; significant differences between before and after 6 months in the exercise group, ${ }^{\mathrm{b}} \mathrm{P}<.05$; significant differences between before and after 12 months in the exercise group, ${ }^{\mathrm{C}} \mathrm{P}<.05$; significant differences between after 6 months and 12 months in the exercise group, ${ }^{d} \mathrm{P}<.05$; significant differences between before and after 6 months in the control group, ${ }^{e} \mathrm{P}<.05$; significant differences between before and after 12 months in the control group, ${ }^{\mathrm{f}} \mathrm{P}<.05$; significant differences between after 6 months and 12 months in the control group, ${ }^{9} \mathrm{P}<.05$; significant differences between the exercise and control groups at after 6 months, $\mathrm{h} \mathrm{P}<.01$; significant differences between the exercise and control groups at after 12 months. 

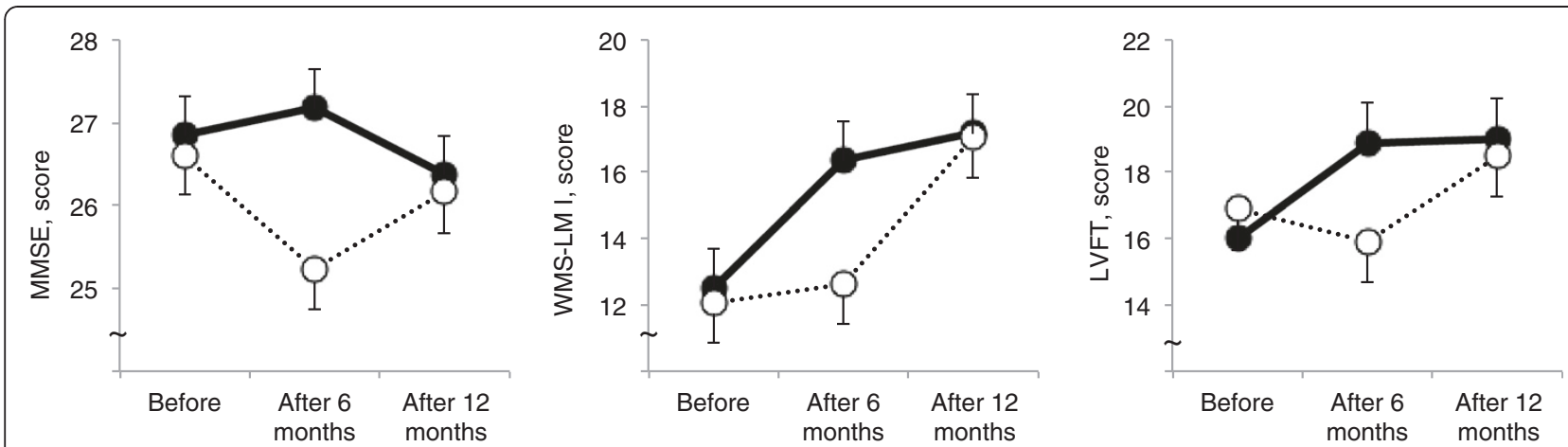

Figure 2 Changes in the MMSE, WMS, and LVFT scores. MMSE; mini-mental state examination, WMS-LM I; Logical Memory I subtest of the Wechsler memory scale-revised, LVFT; letter verbal fluency test. Panels showed change in MMSE, WMS-LM I, and LVFT scores before, after 6 months, and after 12 months intervention. Solid and dashed lines indicate the exercise and control groups, respectively. Group mean and standard errors are shown in older adults with amnestic mild cognitive impairment. The linear mixed models revealed significant group $\times$ time interactions in MMSE $(P=0.04)$, WMS-LM I $(P=0.03)$, and LVFT $(P=0.02)$.

months compared to before intervention $(P<0.05)$ and there was significant differences between the groups at after 6 months $(P<0.05)$.

For the WMS-LM I, there was a group $\times$ time interaction $(P=0.03)$; there were an overall effect of time and no main effect of group (Figure 2). In the post hoc analysis, the exercise group showed significant increase in the WMS-LM I score after 6 and 12 months compared to before intervention $(P<0.05)$ and the control group showed significant increase after 12 months compared to before intervention and after 6 months $(P<0.05)$. There was a significant difference between the groups at 6 months $(P<0.05)$.

On the LVFT, there was a group $\times$ time interaction $(P=0.02)$; there were an overall effect of time and no main effect of group (Figure 2). In the post hoc analysis, There were no significant differences between the times and groups.

The WMS-LM II, DSC, and SCWT-I showed main effect of time, although there were no group $\times$ time interaction and main effect of group. In the post hoc analysis, the exercise group showed significant increase in the WMS-LM II score after 6 and 12 months compared to before intervention $(P<0.05)$ and the control group showed significant increase after 12 months compared to before intervention and after 6 months $(P<0.05)$. There were no significant differences between the groups at each timepoints (Table 2).

\section{Discussion}

There was a significant group $\times$ time interaction on the MMSE, WMS-LM I, and LVFT scores. Twelve months of multicomponent exercise improved cognitive function in older adults with aMCI relative to the education control group. In particular, positive effects were observed for general cognitive function, immediate memory, and language ability, which is consistent with findings in cognitively intact adults [28]. A recent randomized controlled trial has been described as providing verification of the benefits of exercise in elderly adults with MCI [23]. In that study, 152 participants were randomly assigned to an aerobic exercise group and a non-aerobic exercise group, and to a vitamin B group and a placebo group, and a one-year intervention was carried out. The participants exercised twice weekly for 60 minutes each time. For the aerobic exercises, they walked together in groups. The results showed that aerobic exercise has no significant effect in improving cognitive function. However, these results were based on an intention-to-treat analysis, which included 30 participants who did not attend the exercise sessions. Had those elderly adults who had a high attendance rate among the aerobic exercise group been included in the analysis, then the results would have shown increased memory and attention, confirming the effectiveness of aerobic exercise in elder adults with MCI, though only to a limited extent. In another recent report, when elderly adults with MCI (a mean age of 70 years) engaged in aerobic exercise four times every week over the course of six months with a heart rate reserve of $75 \%$ to $85 \%$, executive function significantly improved [16].

The present study shows that significant interactions were observed in general cognitive function, immediate memory, and verbal fluency between the groups, although intervention effects on delayed memory, processing speed, and executive control did not reach significance. Lautenschlager et al. reported that physical activity and behavioral interventions improve general cognitive function [19]. The multicomponent exercise training used in the current study also included aerobic exercise and behavioral interventions, such as selfmonitoring of home-based exercise. Our results further 
supported the idea that a composite approach including aerobic exercise and behavioral interventions can have beneficial effects on cognitive function in aMCI patients.

Older adults with aMCI exhibit greater decreases in memory function than in other cognitive functions, relative to healthy older adults [40]. The cognitive deficits in aMCI increase the risk of conversion from MCI to $\mathrm{AD}$ $[11,12]$. Enhancing cognitive function, especially memory, in $\mathrm{MCI}$ may prevent conversion from $\mathrm{MCI}$ to $\mathrm{AD}$ in older adults. Our multicomponent exercise program involved cognitive loads during exercise. In other words, exercise was conducted under multitask conditions such as dual-task stimulation or while learning tasks during the exercises [41]. Our multicomponent exercise program, involving aerobic exercise, muscle strength, and additional cognitive demand, has some advantages for improving cognitive function over aerobic exercise alone, including possibly increasing logical memory in older adults with aMCI. The WMS-LM I scores in the education control group increased significantly at 12 months compared to before and after 6 months. The education control group received reports of the results of the three assessments and lectures regarding health. We suggest that these educational approaches may be useful in maintaining healthy behavior, such as starting cognitive training or intellectual activities. In fact, the subjects in the control group had fewer cessations of intellectual activity, e.g. culture lessons, than the exercise group during the 12 -month period (-9\% vs. $-19 \%)$.

Baker et al. reported that high intensity aerobic exercise increased VFT scores in older women with MCI [16]. Early in the dementia process, the ability to consciously access lexical information about a target word is impaired while the overall semantic system is intact [42], whereas later in the disease, the integrity of the entire system is compromised, resulting in impaired name recall in structured tasks and spontaneous conversation $[42,43]$. Fluency tests tap into lexical and semantic retrieval operations and may be able to measure these specific aspects of language breakdown in aMCI patients. In a functional neuroimaging study using near infrared spectroscopy, patients with AD showed decreased brain activation patterns compared with healthy controls during the conduct of VFT. Significant correlations between brain activation and performance in the LVFT for dementia patients were found [44]. In the present study, multicomponent exercise provided positive effects on LVFT scores in the aMCI subjects, who had a higher risk of dementia [45].

The present study has several limitations. The small sample size means that replication with a larger group of adults with MCI would be beneficial. Other limitations include unknown group differences in the risk factors of cognitive decline and $\mathrm{AD}$, such as apolipoprotein $\mathrm{E} \varepsilon 4$ genotypes [46], and inflammation [47], although there were no significant differences between the groups in hypertension, diabetes mellitus, medications, biomarkers of lipid metabolism, physical performance, instrumental ADL functioning, and depressive moods. In addition, it is possible that the improvement in the exercise group resulted from the social contact that the intervention group received. This possibility cannot be completely excluded with the present design and should be addressed in future studies.

\section{Conclusions}

Twelve months of exercise improved cognitive function in older adults with aMCI relative to the education control group. In particular, positive effects were observed for general cognitive function, immediate memory, and language ability. A future follow-up investigation is required to determine whether the effect is associated with prevention or delayed onset of dementia in older adults with aMCI.

\section{Abbreviations}

aMCl: Amnestic mild cognitive impairment; AD: Alzheimer's dementia; CDR: Clinical dementia rating; WMS-LM: Wechsler memory scale-logical memory; ADL: Activities of daily living; CONSORT: Consolidated standards of reporting trials; MMSE: Mini-mental state examination; DSC: Digit symbolcoding; LVFT: Letter verbal fluency test; CVFT: Category verbal fluency test; SCWT: Stroop color and word test; ITT: Intention-to-treat.

\section{Competing interests}

The authors declare that they have no competing interests.

\section{Authors' contributions}

Conception of the idea for the study: TS and HS. Development of the protocol and organization: TS, HS, HM, TD, and DY. Acquisition of participants, study management, and statistical analysis: HS, HM, TD, DY, KT, $Y A, K U, S L$, and HP. All authors contributed to the interpretation of the data and drafting the article and provided final approval of the version to be published. All authors read and approved the final manuscript.

\section{Acknowledgements}

We would like to thank the Obu city office for the help provided with participant recruitment, the speech therapists of the Ukai rehabilitation hospital, and the radiological technologists of the National Center for Geriatrics and Gerontology for their assistance with data collection. This study was supported in part by a grant from the Japanese Ministry of Health, Labour and Welfare [Project for optimizing long-term care; B-3] and a Grantin-Aid for Scientific Research from the Ministry of Education and Culture of Japan. No support was received from industry. All authors declare that they have no competing interests and have nothing to declare. The researchers were all independent from the funder. The sponsors had no role in the design or conduct of the study, the collection, management, analysis, or interpretation of the data, or the preparation, review, or approval of the manuscript.

\section{Author details}

${ }^{1}$ Research Institute, National Center for Geriatrics and Gerontology, Obu, Japan. ${ }^{2}$ Section for Health Promotion, Department for Research and Development to Support Independent Life of Elderly, Center for Gerontology and Social Science, National Center for Geriatrics and Gerontology, Obu, Japan. ${ }^{3}$ Section for Physical Functioning Activation, Department of Functioning Activation, Center for Gerontology and Social Science, National Center for Geriatrics and Gerontology, Obu, Japan. 
Received: 23 March 2012 Accepted: 10 October 2012

Published: 31 October 2012

\section{References}

1. Ganguli M, Dodge HH, Shen C, DeKosky ST: Mild cognitive impairment, amnestic type: an epidemiologic study. Neurology 2004, 63(1):115-121.

2. Lopez OL, Jagust WJ, DeKosky ST, Becker JT, Fitzpatrick A, Dulberg C, Breitner J, Lyketsos C, Jones B, Kawas C, et al: Prevalence and classification of mild cognitive impairment in the Cardiovascular Health Study Cognition Study: part 1. Arch Neurol 2003, 60(10):1385-1389.

3. Ritchie K, Artero S, Touchon J: Classification criteria for mild cognitive impairment: a population-based validation study. Neurology 2001, 56(1):37-42

4. Di Carlo A, Baldereschi M, Amaducci L, Maggi S, Grigoletto F, Scarlato G, Inzitari D: Cognitive impairment without dementia in older people: prevalence, vascular risk factors, impact on disability. The Italian Longitudinal Study on Aging. J Am Geriatr Soc 2000, 48(7):775-782.

5. Graham JE, Rockwood K, Beattie BL, Eastwood R, Gauthier S, Tuokko H, McDowell I: Prevalence and severity of cognitive impairment with and without dementia in an elderly population. Lancet 1997, 349(9068):1793-1796.

6. Manly JJ, Bell-McGinty S, Tang MX, Schupf N, Stern Y, Mayeux R: Implementing diagnostic criteria and estimating frequency of mild cognitive impairment in an urban community. Arch Neurol 2005, 62(11):1739-1746.

7. Petersen RC, Doody R, Kurz A, Mohs RC, Morris JC, Rabins PV, Ritchie K, Rossor M, Thal L, Winblad B: Current concepts in mild cognitive impairment. Arch Neurol 2001, 58(12):1985-1992.

8. Palmer K, Wang HX, Backman L, Winblad B, Fratiglioni L: Differential evolution of cognitive impairment in nondemented older persons: results from the Kungsholmen Project. Am J Psychiatry 2002, 159(3):436-442.

9. Winblad B, Palmer K, Kivipelto M, Jelic V, Fratiglioni L, Wahlund LO, Nordberg A, Backman L, Albert M, Almkvist O, et al: Mild cognitive impairment-beyond controversies, towards a consensus: report of the International Working Group on Mild Cognitive Impairment. J Intern Med 2004, 256(3):240-246.

10. Winblad B, Wimo A, Engedal K, Soininen H, Verhey F, Waldemar G, Wetterholm AL, Haglund A, Zhang R, Schindler R: 3-year study of donepezil therapy in Alzheimer's disease: effects of early and continuous therapy. Dement Geriatr Cogn Disord 2006, 21(5-6):353-363.

11. Amieva H, Letenneur L, Dartigues JF, Rouch-Leroyer I, Sourgen C, D'AlcheeBiree F, Dib M, Barberger-Gateau P, Orgogozo JM, Fabrigoule C: Annual rate and predictors of conversion to dementia in subjects presenting mild cognitive impairment criteria defined according to a population-based study. Dement Geriatr Cogn Disord 2004, 18(1):87-93.

12. Tabert MH, Manly JJ, Liu X, Pelton GH, Rosenblum S, Jacobs M, Zamora D, Goodkind M, Bell K, Stern Y, et al: Neuropsychological prediction of conversion to Alzheimer disease in patients with mild cognitive impairment. Arch Gen Psychiatry 2006, 63(8):916-924

13. Chen P, Ratcliff G, Belle SH, Cauley JA, DeKosky ST, Ganguli M: Cognitive tests that best discriminate between presymptomatic $A D$ and those who remain nondemented. Neurology 2000, 55(12):1847-1853.

14. Visser PJ, Verhey FR, Hofman PA, Scheltens P, Jolles J: Medial temporal lobe atrophy predicts Alzheimer's disease in patients with minor cognitive impairment. J Neurol Neurosurg Psychiatry 2002, 72(4):491-497.

15. Larson EB, Wang L, Bowen JD, McCormick WC, Teri L, Crane P, Kukull W: Exercise is associated with reduced risk for incident dementia among persons 65 years of age and older. Ann Intern Med 2006, 144(2):73-81.

16. Baker LD, Frank LL, Foster-Schubert K, Green PS, Wilkinson CW, McTiernan A, Plymate SR, Fishel MA, Watson GS, Cholerton BA, et al: Effects of aerobic exercise on mild cognitive impairment: a controlled trial. Arch Neurol 2010, 67(1):71-79.

17. Hamer M, Chida Y: Physical activity and risk of neurodegenerative disease: a systematic review of prospective evidence. Psychol Med 2009, 39(1):3-11.

18. Kramer AF, Hahn S, Cohen NJ, Banich MT, McAuley E, Harrison CR, Chason J, Vakil E, Bardell L, Boileau RA, et al: Ageing, fitness and neurocognitive function. Nature 1999, 400(6743):418-419.

19. Lautenschlager NT, Cox KL, Flicker L, Foster JK, Van Bockxmeer FM, Xiao J, Greenop KR, Almeida OP: Effect of physical activity on cognitive function in older adults at risk for Alzheimer disease: a randomized trial. JAMA 2008, 300(9):1027-1037.

20. Masley SC, Weaver W, Peri G, Phillips SE: Efficacy of lifestyle changes in modifying practical markers of wellness and aging. Altern Ther Health Med 2008, 14(2):24-29.
21. Okumiya K, Matsubayashi K, Wada T, Kimura S, Doi Y, Ozawa T: Effects of exercise on neurobehavioral function in community-dwelling older people more than 75 years of age. J Am Geriatr Soc 1996, 44(5):569-572.

22. Scherder EJ, Van Paasschen J, Deijen JB, Van Der Knokke S, Orlebeke JF, Burgers I, Devriese PP, Swaab DF, Sergeant JA: Physical activity and executive functions in the elderly with mild cognitive impairment. Aging Ment Health 2005, 9(3):272-280.

23. van Uffelen JG, Chinapaw MJ, van Mechelen W, Hopman-Rock M: Walking or vitamin B for cognition in older adults with mild cognitive impairment? A randomised controlled trial. Br J Sports Med 2008, 42(5):344-351.

24. Weuve J, Kang JH, Manson JE, Breteler MM, Ware JH, Grodstein F: Physical activity, including walking, and cognitive function in older women. JAMA 2004, 292(12):1454-1461

25. Yaffe $K$, Barnes D, Nevitt M, Lui LY, Covinsky K: A prospective study of physical activity and cognitive decline in elderly women: women who walk. Arch Intern Med 2001, 161(14):1703-1708.

26. Angevaren M, Aufdemkampe G, Verhaar HJ, Aleman A, Vanhees L: Physical activity and enhanced fitness to improve cognitive function in older people without known cognitive impairment. Cochrane Database Syst Rev 2008, (3):CD005381.

27. van Uffelen JG, Chin APMJ, Hopman-Rock M, van Mechelen W: The effects of exercise on cognition in older adults with and without cognitive decline: a systematic review. Clin J Sport Med 2008, 18(6):486-500.

28. Smith PJ, Blumenthal JA, Hoffman BM, Cooper H, Strauman TA, Welsh-Bohmer K, Browndyke JN, Sherwood A: Aerobic exercise and neurocognitive performance: a meta-analytic review of randomized controlled trials. Psychosom Med 2010, 72(3):239-252.

29. Colcombe S, Kramer AF: Fitness effects on the cognitive function of older adults: a meta-analytic study. Psychol Sci 2003, 14(2):125-130.

30. Morris JC: The Clinical Dementia Rating (CDR): current version and scoring rules. Neurology 1993, 43(11):2412-2414.

31. Petersen RC: Mild cognitive impairment as a diagnostic entity. J Intern Med 2004, 256(3):183-194.

32. Wechsler D, Stone C: Wechsler Memory Scale manual. New York: Psychological Corp; 1973.

33. Aisen PS, Petersen RC, Donohue MC, Gamst A, Raman R, Thomas RG, Walter S, Trojanowski JQ, Shaw LM, Beckett LA, et al: Clinical Core of the Alzheimer's Disease Neuroimaging Initiative: progress and plans. Alzheimers Dement 2010, 6(3):239-246.

34. Schulz KF, Altman DG, Moher D: CONSORT 2010 statement: updated guidelines for reporting parallel group randomised trials. BMJ 2010, 340:c332.

35. Folstein MF, Folstein SE, McHugh PR: "Mini-mental state". A practical method for grading the cognitive state of patients for the clinician. J Psychiatr Res 1975, 12(3):189-198.

36. Wechsler D: Wechsler Adult Intelligence Scale-III. San Antonio: The Psychological Corporation; 1997.

37. Nutter-Upham KE, Saykin AJ, Rabin LA, Roth RM, Wishart HA, Pare N, Flashman $L A$ : Verbal fluency performance in amnestic $\mathrm{MCl}$ and older adults with cognitive complaints. Arch Clin Neuropsychol 2008, 23(3):229-241.

38. Lonie JA, Herrmann LL, Tierney KM, Donaghey C, O'Carroll R, Lee A, Ebmeier $\mathrm{KP}$ : Lexical and semantic fluency discrepancy scores in aMCl and early Alzheimer's disease. J Neuropsychol 2009, 3(Pt 1):79-92.

39. Stroop JR: Studies of interference in serial verbal reactions. J Exp Psychol 1935, 18:643-662.

40. Petersen RC, Smith GE, Waring SC, Ivnik RJ, Tangalos EG, Kokmen E: Mild cognitive impairment: clinical characterization and outcome. Arch Neurol 1999, 56(3):303-308

41. Woollacott M, Shumway-Cook A: Attention and the control of posture and gait: a review of an emerging area of research. Gait Posture 2002, 16(1):1-14.

42. Chenery HJ, Murdoch BE, Ingram JCL: An investigation of confrontation naming performance in Alzheimer's dementia as a function of disease severity. Aphasiology 1996, 10:423-441.

43. Nicholas M, Obler LK, Au R, Albert ML: On the nature of naming errors in aging and dementia: a study of semantic relatedness. Brain Lang 1996, 54:184-195.

44. Herrmann MJ, Langer JB, Jacob C, Ehlis AC, Fallgatter AJ: Reduced prefrontal oxygenation in Alzheimer disease during verbal fluency tasks. Am J Geriatr Psychiatry 2008, 16(2):125-135.

45. Gao S, Hendrie HC, Hall KS, Hui S: The relationships between age, sex, and the incidence of dementia and Alzheimer disease: a meta-analysis. Arch Gen Psychiatry 1998, 55(9):809-815. 
46. Hsiung GY, Sadovnick AD, Feldman H: Apolipoprotein E epsilon4 genotype as a risk factor for cognitive decline and dementia: data from the Canadian Study of Health and Aging. CMAJ 2004, 171(8):863-867.

47. Yaffe K, Kanaya A, Lindquist K, Simonsick EM, Harris T, Shorr Rl, Tylavsky FA, Newman AB: The metabolic syndrome, inflammation, and risk of cognitive decline. JAMA 2004, 292(18):2237-2242.

doi:10.1186/1471-2377-12-128

Cite this article as: Suzuki et al: Effects of multicomponent exercise on cognitive function in older adults with amnestic mild cognitive

impairment: a randomized controlled trial. BMC Neurology 2012 12:128.

\section{Submit your next manuscript to BioMed Central and take full advantage of:}

- Convenient online submission

- Thorough peer review

- No space constraints or color figure charges

- Immediate publication on acceptance

- Inclusion in PubMed, CAS, Scopus and Google Scholar

- Research which is freely available for redistribution 